Pacific Journal of Mathematic 


\section{ON TCHEBYCHEFF POLYNOMIALS}

\section{J. L. UllmaN}

1. Introduction. Let $C$ be a closed bounded set having an infinite number of points. There is a unique polynomial $T_{n}(z)$ of degree $n$, and with one as coefficient of $z^{n}$, such that if $P_{n}(z)$ is any other polynomial with the same normalization,

$$
M_{n}=\max _{z \in C}\left|T_{n}(z)\right|<\max _{z \in O}\left|P_{n}(z)\right| .
$$

This is the Tchebycheff polynomial of degree $n$ associated with $C$.

1.1. Assume that $C$ has positive capacity, used throughout to mean logarithmic capacity, and a connected complement $D$. The conductor potential for such $C$ is a real valued function $U(z)$ defined in $D$ with the properties: (1.2) $U(z)$ is harmonic at finite points of $D,(1.3)$ $U(z)-\log |z|$ is regular at infinity and zero there, (1.4) there is a number $\rho>-\infty$ such that $U(z)>\rho$ for $z$ in $D$, (1.5) if $\left\{z_{i}\right\}$ is a convergent sequence of points with limit point on the boundary of $D$, then $\lim U\left(z_{i}\right)=\rho$, except perhaps when the limit point belongs to a subset of the boundary of capacity zero. The function $U(z)$ has a unique representation as a Lebesgue-Stieltjes integral

$$
U(z)=\int \log |z-t| d \mu .
$$

where $\mu$ is a completely additive, positive set function defined for Borel measurable sets, if it is specified that the carrier of $\mu$ consist of boundary points of $D$. [2].

1.2. Fejér [1] proved that the zeros of $T_{n}(z)$ lie in the convex hull $H$ of $C$. The consequence

$$
\left|z_{n i}\right| \leqq R,
$$

where $z_{n i}$ is a zero of $T_{n}(z)$, and $R$ is a finite constant independent of $n$, will be sufficient for later reference. Let

$$
\rho_{n}=\frac{1}{n} \log M_{n} \text {. }
$$

Szegö [3] proved that

Received April 5, 1954, in revised form February 24, 1959. The author has received support, which he gratefully acknowledges, from the O.N.R. contract Nonr-330(00) administered by the University of Michigan. The results of this paper were presented in Abstract 515 appearing in the Bulletin of the American Mathematical Society, Vol. 60, July 1954. 


$$
\lim \rho_{n}=\rho,
$$

where $\rho$ is essentially defined for a set $C$ of positive capacity in $\S 1.1$, and is taken as zero when $C$ has zero capacity. If $C$ does not have a connected complement, $\rho$ is obtained by taking for $D$ in $\$ 1.1$ the unbounded component of the complement of $C$. The above results in conjunction with an argument due to R. Nevanlinna [2, p. 127], can be used to show that

$$
\lim \frac{1}{n} \log \left|T_{n}(z)\right|=U(z),
$$

for $z$ in the complement of $H$. The following results concern the extension of (1.10) to points of $D$ in $H$.

1.3. Summary of results. Let $C$ be a closed, bounded set of positive capacity, and with connected complement $D$. Let $\nu_{n}(S)$ be the total multiplicity of the zeros of $T_{n}(z)$ in the set $S$. If $E$ is a closed subset of $D$, then

$$
\lim \frac{\nu_{n}(E)}{n}=0
$$

and

$$
\lim \int_{E}\left|\frac{1}{n} \log \right| T_{n}(z)|-U(z)| d A=0 .
$$

If $\Gamma$ is a continuously differentiable curve consisting of points of $D$, and with interior denoted by $I(\Gamma)$, then

$$
\lim \frac{\nu_{n} I((\Gamma))}{n}=\mu(I(\Gamma))
$$

The set function $\mu$ is defined by (1.6). In the case $D$ is bounded by a finite number of analytic, Jordan curves, then

$$
\nu_{n}(E)<P \text {, }
$$

where $P$ is a constant depending on $E$, but not on $n$. Also in this case

$$
\lim \frac{1}{n} \log \left|T_{n}(z)\right|=U(z),
$$

for $z$ in $E$, with the possible exception of a set of measure zero.

2. The results concerning the zeros of $T_{n}(z)$, namely (I) and (IV), are established first.

2.1. Lemma 1. Associated with $D$ is a set of domains $\left\{D_{n}\right\}$, 
$n=1,2, \cdots$, with the properties:

(a) $D_{n}$ is an unbounded domain,

(b) the closure of $D_{n}$ is contained in $D_{n+1}$, that is $\bar{D}_{n} \subset D_{n+1}$,

(c) each point of $D$ is contained in some $D_{n}$.

LEMMA 2. Let $u(z)$ be harmonic at finite points of $D$ and regular at infinity. Furthermore, if $\left\{z_{i}\right\}$ is a convergent sequence of points with limit point on the boundary of $D$, suppose that $\lim \inf u\left(z_{i}\right) \geqq 0$, except possibly if the limit point belongs to a subset of the boundary of capacity zero. If, in the exceptional cases, $\lim \inf u\left(z_{i}\right) \geqq-\gamma, 0 \leqq \gamma<\infty$, then in fact $\gamma=0$, and $u(z) \geqq 0$, for $z$ in $D$. [2].

2.2. The generalized Green's function of $D$ with pole at $w, G(z, w)$, where the variable $z$ and the parameter $w$ are points of $D$, has the properties:

(2.1) $G(z, w)>0$,

(2.2) $G(z, w)$ is harmonic in $z$, except if $z=w$, and is regular at infinity,

(2.3) $G(z, w)+\log |z-w|$ is regular when $z=w$,

(2.4) if $\left\{z_{i}\right\}$ is a convergent sequence of points with limit point on the boundary of $D$, then $\lim G\left(z_{i}, w\right)$ exists, and is equal to zero, except perhaps if the limit point belongs to a subset of the boundary of capacity zero, and

(2.5) at the exceptional points $\lim \sup G\left(z_{i}, w\right) \leqq M<\infty$, a constant depending on $w$, but not on $\left\{z_{i}\right\}$. When $w=\infty$,

(2.6) $G(z, \infty)=U(z)-\rho$, and

(2.7) for finite or infinite $w, G(z, w)=G(w, z)$.

2.3. Lemma 3. To each domain $D_{k}$ there is a positive constant $m_{k}$, such that

$$
\rho_{n}-\rho \geqq m_{k} \frac{\nu_{n}\left(D_{k}\right)}{n}
$$

Proof. Let

$$
u_{n}(z)=\frac{1}{n} \log \left|T_{n}(z)\right|,
$$

and let $z_{n 1}, \cdots, z_{n m}, m \leqq n$, be the zeros of $T_{n}(z)$ in $D$. The convention used in listing zeros will be to repeat multiple zeros according to their multiplicity. Consider the function

$$
\begin{aligned}
v_{n}(z)=\left(\rho_{n}-u_{n}(z)\right)+(U(z)-\rho)-\frac{1}{n}\left(G\left(z, z_{n 1}\right)\right. & +\cdots \\
& \left.+G\left(z, z_{n m}\right)\right),
\end{aligned}
$$




$$
=A_{1}(z)+A_{2}(z)-A_{3}(z) .
$$

Let $\left\{z_{i}\right\}$ be a convergent sequence of points of $D$ with limit point on the boundary. Now, $\lim A_{1}\left(z_{i}\right) \geqq 0$ by (1.1), (1.8) and (2.9), $\lim \inf A_{2}\left(z_{i}\right) \geqq 0$ by (1.4), and $\lim A_{3}\left(z_{i}\right)=0$, except possibly if the limit point belongs to a subset of the boundary of capacity zero. In the exceptional case $\lim \sup A_{3}\left(z_{i}\right) \leqq M<\infty$, by (2.5). In addition $v_{n}(z)$ is harmonic in $D$ and regular at infinity. The conditions of Lemma 2 are thus satisfied so that

$$
v_{n}(z) \geqq 0,
$$

for $z$ in $D$. Let $z_{n 1}, \cdots, z_{n p}, p \leqq m$, be the zeros of $T_{n}(z)$ in $D_{k}$. Then, by (2.1), (2.7), (2.10), (2.12),

$$
\rho_{n}-\rho-\left(u_{n}(z)-U(z)\right) \geqq \frac{1}{n}\left(G\left(z_{n 1}, z\right)+\cdots+G\left(z_{n p}, z\right)\right) .
$$

If $m_{k}$ is the lower bound of $G(z, \infty)$ on $D_{k}$, then the value of $(2.13)$ at $z=\infty$ yields (2.8).

2.4. Proof of (I). The set $E$ will be contained in an element of $\left\{D_{n}\right\}$, say $D_{k}$. Hence by (2.8) and the definition of $\nu_{n}(S)$,

$$
\frac{\nu_{n}(E)}{n} \leqq \frac{\nu_{n}\left(D_{k}\right)}{n} \leqq \frac{\rho_{n}-\rho}{m_{k}} .
$$

The result then follows by (1.9).

2.5. Proof of (IV). Szegö [4] has shown, under the added restriction on $D$, that

$$
\rho_{n}-\rho \leqq \frac{K}{n}
$$

where $K$ is a constant not depending on $n$. This together with (2.8) yields

$$
\nu_{n}\left(D_{k}\right) \leqq \frac{K}{m_{k}} .
$$

Thus if $D_{k}$ contains $E$, the assertion follows.

3. The next results proved are (II) and (V) concerning the mean convergence in the general case, and the point wise convergence in a special case, of the sequence $u_{n}(z)=1 / n \log \left|T_{n}(z)\right|$.

3.1. Let $D_{k}$ again be a domain containing $E$. Assign to each point of $E$ a circle centered at the point, lying in $D_{k}$, and with radius not 
exceeding 1/3. By the Heine-Borel theorem, a finite number of circles cover $E$. Hence it is sufficient to prove (II), replacing $E$ by a circle in $D_{k}$ with radius less than $1 / 3$.

3.2. Let $s_{n 1}, \cdots, s_{n n_{1}}$ be the zeros of $T_{n}(z)$ in the complement of $D_{k+1}$ and let $r_{n 1}, \cdots, r_{n n_{2}}$ be the zeros in $D_{k+1}$. By the convention of listing multiple zeros, $n_{1}+n_{2}=n$. Note that by (I),

$$
\lim \frac{n_{2}}{n}=0 \text {. }
$$

Next define

$$
S_{n}(z)=\prod_{i-1}^{n_{1}}\left(z-s_{n \ell}\right)
$$

and

$$
R_{n}(z)=\prod_{i=1}^{n_{2}}\left(z-r_{n i}\right)
$$

Now

$$
\begin{aligned}
& \left|\frac{1}{n} \log \right| T_{n}(z)|-U(z)| \\
= & \left|\frac{n_{1}}{n} \frac{1}{n_{1}} \log \right| S_{n}(z)\left|+\frac{1}{n} \log \right| R_{n}(z)|-U(z)| \\
\leqq & \frac{n_{1}}{n}\left|\frac{1}{n_{1}} \log \right| S_{n}(z)|-U(z)|+\frac{n_{2}}{n}|U(z)|+\frac{1}{n}|\log | R_{n}(z) \mid .
\end{aligned}
$$

It will be shown in $\S 4.3$ that the first term of (3.6) tends to zero uniformly in $E$. Also in $E,|U(z)|$ has a finite upper bound, so by (3.1), the second term also tends uniformly to zero in $E$.

3.3. Proof of (II). By the remarks of $\S \S 3.1$ and 3.2 , it is sufficient to prove

$$
\lim \frac{1}{n} \int_{|z-a|<\delta} \log \left|R_{n}(z)\right| d A_{z}=0,
$$

where $|z-a|<\delta$ is a subset of $D_{k}$ and $\delta \leqq 1 / 3$. Let

$$
\frac{1}{n} \log \left|R_{n}(z)\right|=\int \log |z-t| d \mu_{n} .
$$

The integral in (3.7) then has the upper bound

$$
\begin{aligned}
& \int_{|z-a|<\delta,}\left|\int_{|t-a|<2 \delta} \log \right| z-t\left|d \mu_{n}\right| d A_{z} \\
& \quad+\int_{|z-a|<\delta,}\left|\int_{|t-a| \geqq 2 \delta} \log \right| z-t\left|d \mu_{n}\right| d A_{z} .
\end{aligned}
$$


By (1.7) $\mu_{n}(S)$ is zero for any set $S$ in the exterior of $|z|=R$. Hence the second integral in (3.9) is bounded by

$$
\pi \delta^{2} \frac{n_{2}}{n} \max \{|\log | R+\delta\|,|\log | \delta\|\} .
$$

This tends to zero by (3.1). The first integral can be written

$$
\int_{|z-a|<\delta}\left(\int_{|b-a|<2 \delta} \log \frac{1}{|z-t|} d \mu_{n}\right) d A_{z},
$$

since

$$
|z-t| \leqq|z-a|+|t-a|<3 \delta \leqq 1 .
$$

The order of integration can be changed, to yield

$$
\int_{|t-a|<2 \delta|z-a|<\delta}\left(\int \log \frac{1}{|z-t|} d A_{z}\right) d \mu_{n},
$$

or

$$
\int_{|t-a|<2 \delta} g(t) d \mu_{n}
$$

where

$$
g(t)=\left\{\begin{array}{l}
\pi \delta^{2} \log \frac{1}{|t-a|}, \quad \delta \leqq|t-a|<2 \delta, \\
\pi \delta^{2} \log \frac{1}{\delta}+\frac{\pi}{2}\left(\delta^{2}-|t-a|^{2}\right), \quad 0 \leqq|t-a|<\delta .
\end{array}\right.
$$

From this it follows that an upper bound for (3.11) is

$$
\frac{n_{2}}{n} g(a)
$$

This tends to zero by (3.1).

3.4. Proof of (V). The contents of $\S 3.2$, in particular (3.6), reduce the proof to showing

$$
\lim \frac{1}{n} \log \left|R_{n}(z)\right|=0,
$$

for $z$ in $E$, except possibly for a set of measure zero. Fy (IV) there are less than $P$ zeros in $E$ for each $n$, and each of these, by (1.7) is inside or on the circle $|z|=R$. Hence it is sufficient to show

$$
\lim r_{n}(z)=\lim \frac{1}{n}|\log | z-a_{n}||=0,
$$


where $\left|a_{n}\right| \leqq R$, for $|z|<Q$, a disc covering $E$, with the possible exception of a set $T$ of measure zero. For a fixed integer $k>0$,

$$
r_{n}(z)>\frac{1}{k}
$$

either if

$$
\left|z-a_{n}\right|>\exp \left(\frac{n}{k}\right)
$$

or

$$
\left|z-a_{n}\right|<\exp \left(-\frac{n}{k}\right)
$$

Now (3.20) will ultimately fail to hold since $\left|z-a_{n}\right| \leqq R+Q$. Let $T(k)$ be the set of $z$ for which (3.21) holds infinitely often, and let $T(k, p)$ be the set where (3.21) holds for some $n \geqq p$. It is clear that

$$
T(k) \subset T(k, p) \text {. }
$$

Hence if $m_{e}(S)$ designates the exterior measure of a set $S$,

$$
\begin{gathered}
m_{e}(T(k)) \leqq m_{e}(T(k, p)) \leqq \pi \sum_{n=p}^{\infty} \exp \left(\frac{-2 n}{k}\right) \\
=\exp \left(\frac{-2 p}{k}\right)\left(1-\exp \left(\frac{-2}{k}\right)\right)^{-1} .
\end{gathered}
$$

This bound holds for all values of $p$. Thus the exterior measure of $T(k)$, and hence its measure, is zero. Since $T$ is the set where

$$
\lim \sup r_{n}(z)>0 \text {, }
$$

each point of $T$ is contained in one of the sets $T(k)$. There are a denumerable number of the latter, each having measure zero. $T$ thus has measure zero.

\section{Let}

$$
s_{n}(z)=\frac{1}{n_{1}} \log \left|S_{n}(z)\right|
$$

It is first shown that

$$
\lim s_{n}(z)=U(z)
$$

for $z$ in $D_{k+1}$, and that the convergence is uniform in $D_{k}$. This result completes the argument based on (3.6). The divergence theorem is then applied to (4.2) to yield the proof of (III). 
4.1. Lemma 4. If

$$
\sigma_{n}=\max _{z \in O} s_{n}(z)
$$

then

$$
\lim \sigma_{n}=\rho .
$$

Proof. By (1.1), (1.8), (2.9), (4.3),

$$
\sigma_{n}=\max s_{n}(z) \geqq \max u_{n_{1}}(z)=\rho_{n_{1}} .
$$

Let $z_{1}$ be a point of $C$ for which

$$
\sigma_{n}=s_{n}\left(z_{1}\right) \text {. }
$$

Then

$$
\begin{aligned}
\rho_{n} \geqq u_{n}\left(z_{1}\right) & =\frac{n_{1}}{n} s_{n}\left(z_{1}\right)+\frac{1}{n} \log \left|R_{n}\left(z_{1}\right)\right|, \\
& =\frac{n_{1}}{n} \sigma_{n}+\frac{1}{n} \log \left|R_{n}\left(z_{1}\right)\right| .
\end{aligned}
$$

Now $z_{1}$ is bounded from $D_{k+1}$, the domain containing the $r_{n i}$, and $\left|r_{n i}\right|$ has a bound independent of $n$ by (1.7). Hence there are positive constants, $a$ and $b$, such that

$$
0<a \leqq\left|z_{1}-r_{n i}\right| \leqq b<\infty,
$$

for all $n$ and $i$. Combining this with (3.3) and (4.7) yields

$$
\rho_{n} \geqq \frac{n_{1}}{n} \sigma_{n}-\frac{n_{2}}{n} K,
$$

where $K=\max \{|\log a|,|\log b|\}$. From this and (4.5) it then follows that

$$
\rho_{n_{1}} \leqq \sigma_{n} \leqq \frac{n}{n_{1}} \rho_{n}+\frac{n_{2}}{n_{1}} K
$$

The conclusion of the lemma now follows by (1.9), (3.1).

4.2. Form the function

$$
w_{n}(z)=\sigma_{n}-s_{n}(z)-(\rho-U(z)) .
$$

This can be treated like $v_{n}(z),(2.10)$, to show that it is positive in $D$.

Lemma 5. The functions $w_{n}(z)$ converge to zero in $D_{k+1}$, and uniformly in $\bar{D}_{k}$.

Proof. Let the disc $|z-a| \leqq \gamma$ lie in $D_{k+1}$, and let $z_{1}=a+r \exp (i \theta)$, 
$r \leqq s<\gamma$. Since $w_{n}(z)$ is positive in $D_{k+1}$, and clearly harmonic there, the inequality

$$
\frac{\gamma-s}{\gamma+s} w_{n}(a) \leqq w_{n}\left(z_{1}\right) \leqq \frac{\gamma+s}{\gamma-s} w_{n}(a)
$$

holds. This shows that the convergence of $w_{n}(a)$ to zero implies the uniform convergence to zero in the circle $|z-a|=s$, and that if $w_{n}(a)$ does not converge to zero, the same will be true at each point of the circle. A similar relationship holds between the convergence of $w_{n}(\infty)$ and the convergence of $w_{n}(z)$ for $|z| \geqq s$, a domain lying in $D_{k+1}$. Thus the set of points of $D_{k+1}$ where $\lim w_{n}(z)=0$ is an open set, and the set where $\lim w_{n}(z) \neq 0$ is also an open set. Since $D_{k+1}$ is open and connected, it cannot be expressed as the sum of two disjoint open sets, so that one of these sets must be a null set. Since $w_{n}(\infty)=\sigma_{n}-\rho$, a quantity tending to zero by Lemma 4 , the non-null set is the one for which $\lim w_{n}(z)=0$. By the Heine-Borel theorem, $\bar{D}_{k}$ can be covered by a finite number of circles lying in $D_{k+1}$, one of which will be of the form $|z| \geqq s$. The convergence will be uniform in each circle, and hence uniform in $\bar{D}_{k}$.

4.3. For application to (3.6), note that

$$
\left|\frac{1}{n_{1}} \log S_{n}(z)-U(z)\right| \leqq\left|w_{n}(z)\right|+\left|\sigma_{n}-\rho\right| \text {. }
$$

Thus by Lemmas 4 and 5 , the left side converges uniformly to zero in $\bar{D}_{k}$, and hence in $E$.

4.4. Proof of (III). There is no loss in generality in assuming that $\Gamma$ lies in $D_{k}$. If $z=a+r \exp (i \theta), r \leqq s<\gamma$, then

$$
\left|\left(w_{n}(z)\right)_{x}\right| \leqq \frac{w_{n}(a)}{(\gamma-s)^{2}}
$$

where ()$_{x}$ denotes the partial derivative with respect to $x$. It is assumed that $a$ is on $\Gamma$, and that $|z-a| \leqq s$ lies in $D_{k+1}$. The same inequality holds for the partial derivative with respect to $y$. The convergence of $w_{n}(a)$ to zero thus yields the uniform convergence to zero of the partial derivatives in the specified circles. An application of the Heine-Borel theorem then shows that the convergence is uniform on $\Gamma$. Thus

$$
\lim \frac{1}{2 \pi} \int_{r^{\prime}}\left(w_{n}(z)\right)_{x} d y-\left(w_{n}(z)\right)_{y} d x=0 .
$$

Using (4.11), it is seen that this is equivalent to 


$$
\begin{aligned}
& \lim \frac{1}{2 \pi} \int_{I^{\prime}}\left(s_{n}(z)\right)_{x} d y-\left(s_{n}(z)\right)_{y} d x \\
& \quad=\frac{1}{2 \pi} \int(U(z))_{x} d y-(U(z))_{y} d x
\end{aligned}
$$

Let $\lambda_{n}(S)$ be the total multiplicity of the zeros of $S_{n}(z)$ in the set $S$. Now both $U(z)$ and $s_{n}(z)$ are harmonic on $\Gamma$, and $\Gamma$ is of sufficient smoothness for the application of the divergence theorem, so that the result

$$
\lim \frac{\lambda_{n}\left(I\left(\Gamma^{\prime}\right)\right)}{n}=\mu\left(I\left(I^{\prime}\right)\right)
$$

is obtained. For any set $S$ it follows from (3.2) that

$$
\nu_{n}(S)-\lambda_{n}(S) \leqq \nu_{n}\left(D_{k+1}\right)=n_{2} .
$$

Thus, by (3.1) and (4.7) applied to

$$
\frac{\left.\lambda_{n}\left(I \Gamma^{\prime}\right)\right)}{n} \leqq \frac{\nu_{n}\left(I\left(\Gamma^{\prime}\right)\right)}{n} \leqq \frac{\lambda_{n}\left(I\left(I^{\prime}\right)\right)}{n}+\frac{n_{2}}{n},
$$

the proof of (III) is completed.

5. Relationship to a paper by Walsh and Evans. The results (I) and (III) we obtained by other methods in [7], and another form of discussing the asymptotic behavior of $T_{n}(z)$ for $z$ in the complement of $C$ was used. The result (IV) is not found in [7], and we will discuss in more detail, and in a slightly more general context the significance of this and the other results.

Domain Polynomials. Besides the $T_{n}(z)$, there are other sets of polynomials which are associated with general sets $C$ in the plane. We mention only the Carleman polynomials [3], $C_{n}(z)$, which require that $C$ have connected complement, and Faber polynomials [5], $F_{n}(z)$, which require that the complement of $C$ be simply connected. These are adequate to illustrate our remarks.

The Location Problem is an apt name to give to results relating to the location of zeros of domain polynomials, and known results suggest the further distinction of interior location and exterior location, corresponding to whether we refer to zeros on $C$ or in the complement of $C$.

Results on Exterior Location. For sets with simply connected complements, and bounded by a simple analytic curve $\Gamma$, it has been shown by Johnston [3] and the author [5] that ultimately the zeros of $C_{n}(z)$ and $F_{n}(z)$, respectively, lie inside any simple interior level curve of $\Gamma$. It is not known whether this is true for $T_{n}(z)$, although (IV) shows that the zeros lie ultimately inside any exterior level curve. 
A basic observation of this paper and [7] is that when $C$ has a multiply connected complement, then zeros of $T_{n}(z)$ can lie in the complement of $C$ and be uniformly bounded from $C$ for arbitrarily large $n$. In the sense defined by (I) the number must be small in comparison with $n$, although they can exceed any finite bound. The refinement of (IV) states that if $C$ is bounded by a finite number of analytic curves, then there is an absolute constant for any exterior level curve of $C$, which ultimately cannot be exceeded by the number of zeros of $T_{n}(z)$ exterior to this level curve. What has not been shown is whether a constant exists for the complement of $C$ itself. Examples indicate that if there is such a constant, it cannot be less than $k-1$, where $k$ is the number of boundary components of $C$.

Interior Location. Formula (III) states that the proportion of zeros on any component of $C$, for $T_{n}(z)$, approaches the harmonic measure of the component. Where on the component the zeros accumulate is not known. The existant examples, namely $T_{n}(z)$ for the circle and ellipse, indicate that the limit points of the zeros, which can be called the center, have an interior location in the set. No precise characterization of the center for $T_{n}(z)$ has been found. In [6] a study is made of the center for $F_{n}(z)$. The indications are that the center will not be the same set for the different classes of domain polynomials.

\section{BIBLIOGRAPHY}

1. L. Fejer, Über die Lage der Nullstellen von Polynomen, die aus Minimumforderungen gewisser Art entspringen, Mathematische Annalen, 85, (1922), 41-48.

2. Rolf. Nevanlinna, Eindeutige Analytische Functionen, Berlin, 1936.

3. P. C. Rosenbloom and S. E. Warschawski, Approximation by polynomials, Lectures on functions of a complex variable, Edited by Wilfred Kaplan, Ann Arbor, 1955, pp. 287-301.

4. G. Szegö, Bemsrkungen zu einer Arbeit von Herrn Felcete: Über die Verteilung der Wurzeln bei gewissen algebraischen Gleichungen mit ganzzahligen Koeffizienten, Mathematische Zeitschrift, 21 (1924), 203-208.

5. J. L. Ullman, Studies on faber polynomials, Dissertation, Stanford University, 1949.

6. - On faber polynomials, submitted to the Transactions of the Amer. Math. Soc.

7. J. L. Walsh and J. P. Evans, Note on the distribution of aeros of extremal polynomials, Proc. Nat. Acad. Sci., May 1954, pp. 332-337.

The UNIVERsity of Michigan 



\section{PACIFIC JOURNAL OF MATHEMATICS}

\section{EDITORS}

\section{David Gilbarg}

Stanford University Stanford, California

\section{R. A. Beaumont}

University of Washington

Seattle 5, Washington

\section{A. L. Whiteman}

University of Southern California Los Angeles 7, California

L. J. Paige

University of California

Los Angeles 24, California

\section{ASSOCIATE EDITORS}

E. F. BECKENBACH
C. E. BURGESS
E. HEWITT
A. HORN

A. HORN

\author{
V. GANAPATHY IYER \\ R. D. JAMES \\ M. S. KNEBELMAN \\ L. NACHBIN
}

I. NIVEN

T. G. OSTROM

H. L. ROYDEN

M. M. SCHIFFER
E. G. STRAUS

G. SZEKERES

F. WOLF

K. YOSIDA

\section{SUPPORTING INSTITUTIONS}

\author{
UNIVERSITY OF BRITISH COLUMBIA \\ CALIFORNIA INSTITUTE OF TECHNOLOGY \\ UNIVERSITY OF CALIFORNIA \\ MONTANA STATE UNIVERSITY \\ UNIVERSITY OF NEVADA \\ OREGON STATE COLLEGE \\ UNIVERSITY OF OREGON \\ OSAKA UNIVERSITY \\ UNIVERSITY OF SOUTHERN CALIFORNIA
}

\author{
STANFORD UNIVERSITY \\ UNIVERSITY OF TOKYO \\ UNIVERSITY OF UTAH \\ WASHINGTON STATE COLLEGE \\ UNIVERSITY OF WASHINGTON \\ * * * * \\ AMERICAN MATHEMATICAL SOCIETY \\ CALIFORNIA RESEARCH CORPORATION \\ HUGHES AIRCRAFT COMPANY \\ SPACE TECHNOLOGY LABORATORIES
}

Mathematical papers intended for publication in the Pacific Journal of Mathematics should be typewritten (double spaced), and the author should keep a complete copy. Manuscripts may be sent to any one of the four editors. All other communications to the editors should be addressed to the managing editor, L. J. Paige at the University of California, Los Angeles 24, California.

50 reprints per author of each article are furnished free of charge; additional copies may be obtained at cost in multiples of 50 .

The Pacific Journal of Mathematics is published quarterly, in March, June, September, and December. The price per volume (4 numbers) is $\$ 12.00$; single issues, $\$ 3.50$. Back numbers are available. Special price to individual faculty members of supporting institutions and to individual members of the American Mathematical Society: $\$ 4.00$ per volume; single issues, $\$ 1.25$.

Subscriptions, orders for back numbers, and changes of address should be sent to Pacific Journal of Mathematics, 2120 Oxford Street, Berkeley 4, California.

Printed at Kokusai Bunken Insatsusha (International Academic Printing Co., Ltd.), No. 6, 2-chome, Fujimi-cho, Chiyoda-ku, Tokyo, Japan.

PUBLISHED BY PACIFIC IOURNAL OF MATHEMATICS, A NON-PROFIT CORPORATION

The Supporting Institutions listed above contribute to the cost of publication of this Journal, but they are not owners or publishers and have no responsibility for its content or policies. 


\section{Pacific Journal of Mathematics}

\section{Vol. 9, No. $3 \quad$ July, 1959}

Errett Albert Bishop, A minimal boundary for function algebras . . . . . . . . . . . . 629

John W. Brace, The topology of almost uniform convergence . . . . . . . . . . . . 643

Cecil Edmund Burgess, Chainable continua and indecomposability .......... 653

L. Carlitz, Multiplication formulas for products of Bernoulli and Euler

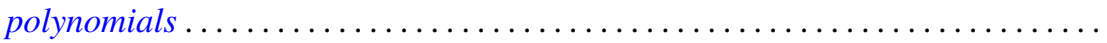

Eckford Cohen, A class of residue systems (mod $r$ ) and related arithmetical

functions. II. Higher dimensional analogues ....................

Shaul Foguel, Boolean algebras of projections of finite multiplicity . . . . . . . . . .

Richard Robinson Goldberg, Averages of Fourier coefficients .................

Seymour Goldberg, Ranges and inverses of perturbed linear operators .

Philip Hartman, On functions representable as a difference of convex functions ....

Milton Vernon Johns, Jr. and Ronald Pyke, On conditional expectation and

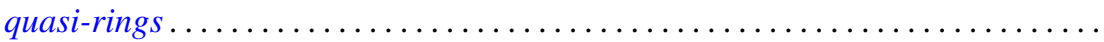

Robert Jacob Koch, Arcs in partially ordered spaces ....................

Gregers Louis Krabbe, A space of multipliers of type $L^{p}(-\infty, \infty) \ldots \ldots \ldots \ldots$

John W. Lamperti and Patrick Colonel Suppes, Chains of infinite order and their

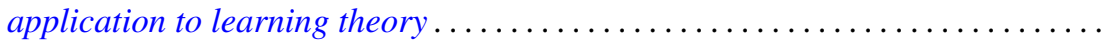

Edith Hirsch Luchins, On radicals and continuity of homomorphisms into Banach

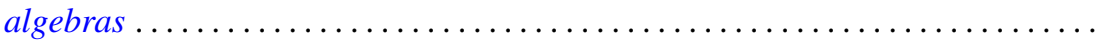

T. M. MacRobert, Multiplication formulae for the E-functions functions of their parameters.

Michael Bahir Maschler, Classes of minimal and representative domains and their kernel functions.

William Schumacher Massey, On the imbeddability of the real projective spaces in Euclidean space.

Thomas Wilson Mullikin, Semi-groups of class $\left(C_{0}\right)$ in $L_{p}$ determined by parabolic differential equations

Steven Orey, Recurrent Markov chains

Ernest Tilden Parker, On quadruply transitive groups ........ . .

Calvin R. Putnam, On Toeplitz matrices, absolute continuity, and unitary

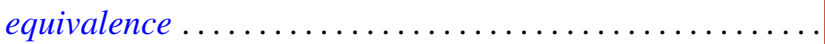

Helmut Heinrich Schaefer, On nonlinear positive operators.

Robert Seall and Marion Wetzel, Some connections between continued fractions and convex sets

Robert Steinberg, Variations on a theme of Chevalley

Olga Taussky and Hans Zassenhaus, On the similarity transformation between a

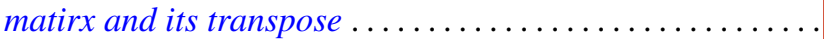

Emery Thomas, The suspension of the generalized Pontrjagin cohomology

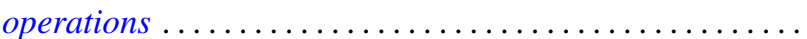

Joseph L. Ullman, On Tchebycheff polynomials ..................... 913

Richard Steven Varga, Orderings of the successive overrelaxation scheme ........ 925

Orlando Eugenio Villamayor, Sr., On weak dimension of algebras . 